CASE 7.-Male, aged 59 years. Oct. 10th, 1912 ; ten months' history. Skiagram showed a stone shadow in the lower end of right ureter. Skiagram showed a stone shadow in the lower end of right ureter.
Cystoscopy showed a large "middle lobe" prostate. Urine clear, no
pus, no albumin. Oct. 24th, 1912, operation, incision as above. Ureter pus, no albumin. Oct. 24 th, 1912, operation, incision as above. Ureter
opened at pelvic brim. Stone lying $2 \frac{1}{2}$ in. below the opening. easily
extracted; ureter closed; rubber drain. No leakage of urine. Wound extracted; ureter closed; rubber drain. No leakage of urine. Wound healed by first intention. Patient left hospital on the fourteenth day;
urine clear at exit. Stone-calcium oxalate, trace of calcium phosphate. urine clear at exit. Stone-calcium oxalate, trace of calcium phosphate.
CASE 8.-Male, aged 46 years; six month' history. Urine contained CASE 8.-Male, aged 46 years; six months' history. Urine contained
fair amount of pus. Skiagram-stone shadow in the left kidney and a fair amount of pus. Skiagram-stone shadow in the left kidney and another in the left lower ureter. March 4th, 1913, left pelvic ureter
exposed as above. As the urine was purulent the ureter was clamped exposed as above. As the urine was purulent the ureter was clamped at the upper end and opened at the lower pelvic brim. Stone extracted with some difficulty by means of a scoop; was lying 3 in. below the
Wound reunited; indiarubber drain. Wound was 21 in. opening. Wound reunited; indiarubber drain. Wound was $2 \frac{1}{2}$ in. long as measured at the end of the operation. First intention. Fight days later nephrolithotomy.

weeks from date of entry 43 years. Jan. 20th, 1913 ; 11 years' history. Urine clear, trace of albumin, calcium oxalate crystals. Skiagramstone shadow in left pelvic ureter and another in the left renal cortex. Jan. 23rd, 1913, operation, incision as above; ureter opened at pelvic brim level. Stone lying 3 in. below opening, easily extracted. Ureter closed; rubber drain: no leakage of urine. First intention wound. Patient got up on thirteenth day, urine being clear. Stone-calcium oxalate. Feb. 13th, 1913, second operation. Left kidney exposed by muscle-splitting incision and stone extracted from the lower middle posterior calyx. No drainage; first intention wound. Patient allowed up on fourteenth day. Urine clear. Stone-calcium oxalate.

CASE 10.-Female, aged 23 years. Two years' history of pain, for which appendix had been removed by another surgeon. Skiagram-stone shadow in left lower ureter. April, 1913, operation. Left ureter exposed through 23 in. incision. Much dilated; opened at pelvic brim, intention. Patient up on ninth day. Stone-calcium oxalate.

CASE 11.-Male, aged 44 years. Oct. 7 th, 1912; four years' history. Urine contained large amount of pus and tubercle bacilli. No radiographic evidence of tuberculosis in the kidneys. The right ureter could be felt per rectum to be thickened, evidence of infection of the right kidney, but not so the left ureter. Several attempts at eystoirritable. Oct. 31 risation of the ureters failed as the bladder was too exposed in the pelvis and found to be adherent to the subperitoneal fat and definitely thickened. This proved that the left kidney was infected as well as the right, and that the case was inoperable. No drainage; first intention. Patient left hospital on twelfth day.

CASE 12.-Male, aged 21 years. August 9th, 1912. Diagnosis-right renal tuberculosis, left kidney healthy and of excellent functioning renal tuberculosis, left kidney healthy and of excellent functioning
powers. August 16th, 1912, operation, Mayo incision, right nephrectomy. Kidney and ureter stuffed with tubercle. A urinary tistula formed in the wound. Sept. 9 th, 1912, second operation, incision as above, right ureterectomy to bladder wall. Ureter cut across with a cautery after it had been ligatured with stout catgut. Wound not

soundly healed.

CASE 13.-Male, aged 37 years. Four months' history; chronic phthisis. Cystoscopic diagnosis-tuberculosis of right kidney. No March 25th, 1913, operation. Left ureter exposed through $2 \frac{1}{2}$ in. March 25th, 1913, operation. Left ureter exposed through $2 \frac{1}{2}$ in. incision; found to be not thickened. Right nephrecton
months later-no symptoms and urine free from pus.

Harley-street, W.

\section{OBSERVATIONS ON "DIAGNOSTIC" TUBERCULIN.}

BY NOEL D. BARDSWELL, M.D. EDIN., M.R.C.P. LOND. \& EDIN., F.R.S.E

MEDICAT SUPERINTENDENT, KIXG EDWARD VII. SANATORIUM, MIDHURST, SUSSEX.

WiTH the introduction of the tuberculin test it was hoped that much of the difficulty attending the recognition of pulmonary tuberculosis in the early stages would be removed, and that in a case presenting signs and symptoms suggestive of tuberculosis, but lacking the definite proof afforded by the presence of tubercle bacilli, the application of the tuberculin test would determine the existence or nonexistence of the disease. This hope has not been fulfilled. Some clinicians, however, are still of the opinion that by the use of tuberculin in such cases a definite diagnosis can be arrived at. Those who hold this view believe that a general reaction-namely, a rise of temperature, malaise, \&c.- to diagnostic doses of tuberculin is sufficient proof of the existence of what may be termed " clinical tuberculosis." If, in addition, there are signs or symptoms suggestive of pulmonary tuberculosis, a positive diagnosis, in their opinion, is justified.

This view unfortunately cannot be maintained. Franz in particular has shown that a very large proportion of apparently healthy men who present no signs or symptoms whatsoever of tuberculosis give a general reaction to diagnostic doses of tuberculin, especially so if the individuals thus examined came from a district in which tuberculosis is prevalent. Such cases Lawrason Brown, of the Adirondack Sanatorium, terms "non-clinical" tuberculosis. It is probable that in such cases there has been at some time an infection with tubercle; the infection, however, has never reached the stage at which it can be diagnosed clinically, is in many cases probably obsolete, and requires nothing in the way of active treatment.

It may be said with confidence that in healthy persons the tuberculin reaction is of no clinical significance, and that in a case of ill-health a reaction to tuberculin is, in itself, no evidence that tuberculosis is the cause of the symptoms. In brief, it is generally recognised that so far as pulmonary tuberculosis is concerned tuberculin for diagnostic purposes has great limitations, since a reaction occurs as readily in cases of mere infection with tuberculosis (non-clinical tuberculosis) as in clinical tuberculosis, and in respect to clinical tuberculosis we are unable to differentiate with any certainty by the use of tuberculin between cases of active. quiescent, and arrested disease. There are, however, good grounds for holding that in the case of a patient presenting indefinite signs and symptoms the failure to react to a subcutaneous dose of 10 milligrammes is strong evidence against the existence of clinical tuberculosis, and that conversely the occurrence of a focal reaction in a suspected area in the lungs is suggestive of the existence of potentially active tubercular disease.

The above represents very briefly the state of our knowledge of the value of tuberculin as a means of diagnosis at the date of the commencement of our work on tuberculin. Since that date (October. 1911) every patient at the sanatorium in whom we have failed to find tubercle bacilli has been subjected to the subcutaneous tuberculin test. In this report I propose to record the results of these observations, and to indicate to what extent we have been influenced by them in the diagnosis and treatment of these particular cases. The number of patients thus observed up to March 31st amounted to 50 .

Method of observation. - The patient (afebrile patients only are tested) is kept at physiological rest for some 48 hours before the inoculations are begun, the temperature being recorded three-hourly throughout this period and during the whole period of subsequent observations.

Dosage.-The sequence of doses used is as follows: First dose, 2/10 milligramme (0.0002 c.c.) albumose-free tuberculin ; second dose, 1 milligramme $(0.001$ c.c. $)$; third doses 5 milligrammes $(0.005 \mathrm{c.c})$; fourth dose, 10 milligramme, (0.01 c.c.).

Site and time of inoculations. - The inoculations are made at $10 \mathrm{~A} . \mathrm{Mr}$, usually in the forearm.

Clinical record.-Note is made daily as to the presence of (a) local reaction at the seat of inoculation; $(b)$ general reaction-namely, rise of temperature, malaise, \&c. ; also of any increase of symptoms, such as cough, sputum, \&c.; and $(c)$ focal reaction-namely, the appearance of, or increase in, physical signs or increase of sputum. Sputum, when available, is carefully tested for tubercle bacilli by the sedimentation method of Ellerman and Erlandson. The 50 cases now under consideration may be for convenience divided in two groups: (1) those in which there were physical signs suggesting the possibility of tuberculosis either in the lung, larynx, or pleura (37 in number); and (2) those in which no physical signs were present (13 in number). It may be assumed that all the 50 cases had something suspicious in their clinical history, symptoms, \&c., inasmuch as they had been sent into the sanatorium as cases of pulmonary tuberculosis. The results of the diagnostic tests are summarised in the following table:-

\begin{tabular}{|c|c|c|c|c|}
\hline - & $\begin{array}{c}\text { Cases with } \\
\text { suspicious } \\
\text { phvsical } \\
\text { signs. }\end{array}$ & $\begin{array}{c}\text { Cases with } \\
\text { no } \\
\text { suspicious } \\
\text { physical } \\
\text { signs. }\end{array}$ & Total. & $\begin{array}{l}\text { Per cent. } \\
\text { of all } \\
\text { cases. }\end{array}$ \\
\hline Gave no reaction ... ... & 8 & 3 & 11 & 22 \\
\hline Gave a focal reaction ... & 12 & 1 & 13 & 26 \\
\hline 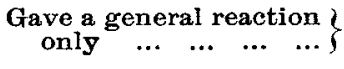 & 17 & 9 & 26 & 52 \\
\hline
\end{tabular}

Indications given by the above Results.

Focal reactions. - The 13 patients who gave a focal reaction were regarded as suffering from tuberculosis in an active or 
potentially active condition. These patients were all retained in the sanatorium.

Negative reactions.-The 11 patients who failed to react to tuberculin were discharged as at least not requiring active treatment in the sanatorium. The 3 patients who had doubtful physical signs were probably cases of non-clinical infection. Of the other 8 patients, some were possibly cases of obsolete disease. In none of these cases was there an association of a clinical history and physical signs strongly suggesting pulmonary tuberculosis. In such a contingency, one that has not yet occurred, we should retain and treat the patient, despite a negative reaction. In 24 cases, therefore, out of 50 , the test gave us information of material value, inasmuch as we were enabled by it to determine that 13 patients certainly required active treatment, and that 11 patients had no occasion to remain longer at the sanatorium.

General reactions only.-In the 26 cases which gave a general reaction, but no focal reaction, the indications were much less definite. As previously stated, the occurrence of a general reaction in itself means no more than that infection with tubercle has taken place at some time. It seems probable that the absence of any focal reaction in such cases, in spite often of very considerable general reaction, gives us positive information of some value. To judge by our experience gained by treating a large number of definitely tuberculous patients with tuberculin a very slight and transient reaction--e.g., a rise of temperature of less than $1^{\circ} \mathrm{F}$. - is very commonly sufficient to increase slightly the amount of sputum for a day when the lesion is active or has been recently active. In such cases, too, an unmistakable focal reaction is, as a rule, very readily produced, and is nearly invariably associated with an increase in the amount of sputum. The absence of any focal reaction or increase of, or appearance of, sputum would seem to be at least suggestive that a tuberculous focus, if existing, is quiescent or obsolete, the indication as to treatment being a careful life rather than anything of a more active character. We have not sufficient experience as yet on which to form any opinion as to how frequently tuberculin in diagnostic doses may fail to produce a focal reaction in cases of active or recently active disease. Again, it is quite conceivable that a focal reaction occurring in a deep-seated lesion may escape detection on physical examination. In some cases, for instance, I have thought that pronounced pleural disease may have masked the physical signs of a focal reaction in the underlying lung. With these various possibilities in mind, we judged each of the 26 cases which gave only a general reaction on its clinical merits, and, from a consideration of the history, symptoms, physical signs, \&c , determined what to us appeared to be the most probable diagnosis. Twelve cases, the most suspicious ones, were treated in the sanatorium for an average period of 12 weeks. Ten cases were treated in the sanatorium for an average period of seven weeks, 3 were discharged after four weeks' observation, and the remaining patient, who was found to be suffering from malignant disease, was discharged as soon as the diagnosis was established.

The practical value of the subcutaneous tuberculin test.-It is impossible to speak with confidence on this point, as we have no means of ascertaining whether the inferences we drew from our series of observations were correct or incorrect. The subsequent after-histories of the cases will, however, give us a very fair answer to the question. Assuming that our judgment has, in the main, been sound, we have been able to form a positive opinion in 48 per cent. of doubtful cases. In the other 52 per cent. we obtained information of a negative character, and had to rely for our diagnosis upon a careful review of all the available clinical data. Our experience, in short, so far, would suggest that tuberculin is of distinct service for diagnostic purposes.

\section{Some Notes as to the Various Reactions.}

The focal reaction. - It is to be noted that the 13 cases which gave a focal reaction reacted in all but one case to a dose less than the maximum, the actual doses being as follows:$0.001,0.0003,0.001,0.005,0.001,0.005,0.01,0.0002$, $0.005,0.001,0.005,0.005,0.005 \mathrm{c.c}$. This supports the view that 0.01 c.c. (10 milligrammes) is an adequate maximum diagnostic dose. In 4 cases a focal reaction occurred comparatively early in the course of the test, and was not associated with any rise of temperatiure or other evidence of a general reaction. There being, therefore, no relation between the intensity of a general reaction and the production of a focal reaction, it is important that a careful clinical examination should be made every day, irrespective of the occurrence of a general reaction. It is clearly undesirable to continue to give tuberculin when a focal reaction has been produced.

The general reaction. - The degree of fever auring a general reaction varies very much ; in our 50 cases from a minimum of $99 \cdot 6^{\circ} \mathrm{F}$. to a maximum of $104 \cdot 2^{\circ}$. A temperature between $101^{\circ}$ and $102^{\circ}$ represents an average reaction. In 25.7 per cent. of those cases a general reaction and a focal reaction were associated.

The relation of these two reactions in 50 cases is shown by the following analysis: Focal reaction with general reaction, 18 per cent. ; focal reaction alone, 8 per cent.; general reaction alone, 52 per cent.; no reaction, 22 per cent. Otten, quoted by Riviere and Morland, gives the following figures for a similar analysis of 324 cases: Focal reaction with general reaction, 60.8 per cent. focal reaction alone, $7 \cdot 4$ per cent.; general reaction alone, 23.5 per cent.; no reaction, 8.3 per cent. Lawrason Brown obtained a focal reaction in some 33 per cent. of 268 cases examined. Bandelier and Roepke, who have had great experience, state that a focal reaction is obtained in one-third of all cases. These figures may be thus compared:-Gave a focal reaction: Otten, 68.2 per cent.; Lawrason Brown, 33 per cent.; Bandelier and Roepke, 33 per cent.; our cases, 26 per cent.

The discrepancy between these sets of figures is noticeable. Our experience is as yet small, but so far as it goes it approximates much more nearly to that of Brown and Bandelier and Roepke than to that of Otten. The figures of these last-named observers are, however, comparable. I think we may assume with a great degree of probability that the wide difference between the experience of Otten and that of Brown and Bandelier and his colleague is due to a varying conception of what constitutes a focal reaction. If the development, or increase, of symptoms referable to the respiratory system, without any increase in physical signs or increase of sputum, is accepted as sufficient evidence of a focal reaction, the proportion of positive focal reactions obtained in a series of cases will be high. In practice, however, it is very difficult to distinguish such symptoms which may possibly represent the focal reaction from those which are the outcome solely of the high fever and constitutional disturbance resulting from the general reaction. In this difficulty lies great scope for fallacy, and for differences of opinion and results.

The appearance of tubercle bacilli in the sputum during a reaction. - In two of our cases tubercle bacilli were found in the sputum during a reaction, though repeated examinations previously had given a negative result. In one of these cases there was no obvious focal reaction and no increase of sputum. In both cases the tubercle bacilli disappeared again from the sputum when the reaction passed off, and in neither case have they as yet been found again. Such an appearance of tubercle bacilli during a reaction is, according to Lawrason Brown, so exceptional, only occurring in his experience in 6 per cent. of 286 cases, that he is of opinion that when it occurs the apparent connexion is merely a coincidence. Bandelier expresses a very similar view. Personally, I cannot think that the appearance of tubercle bacilli in our two cases was simply a coincidence. It would seem more probable that it was the result of the hyperæmia set up in the diseased areas by the reaction. A very comparable experience seems to favour this view. We have on several occasions observed the reappearance of tubercle bacilli in the sputum during the course of an inter-current catarrh. This we attributed to a transient hyperæmia of the quiescent focus of disease resulting from the catarrh. In these cases, also, the tubercle bacilli disappeared again when the catarrh subsided.

The safety of suboutaneous diagnostio inooulations. - As yet we have seen no ill-effects of the result of diagnostic inoculations. The reaction is often very unpleasant to the patient when at its height, but the signs and symptoms produced, whether general or focal, are very transient, and usually pass off completely in from 24 to 48 hours.

The effect of the introduction of the tuberoulin test upon the administration of the sanatorium. - As may readily be expected, the use of "diagnostic tuberculin" is reflected in 
the work of the sanatorium. A certain number of negative eases are discharged after some four weeks' observation, and a somewhat larger proportion of more suspicious cases are retained for some eight to ten weeks only, instead of the usual average of 16 weeks or more. The effect of this is to increase the proportion of patients in the sanatorium who have unmistakable evidence of pulmonary tuberculosisnamely, tubercle bacilli in the sputum.

Midhurst, Sussex.

\section{THE SIGNIFICANCE OF RENAL TUBE CASTS IN THE URINARY SEDIMENT. ${ }^{1}$}

\section{By G. LESTOCK THORNTON, M.A. CANTAB.,} M.R.C.P. LoNo., M.R.C.S. ENG.,

TOYORARY MEDICAL OFFICER TO THE EXMOUTH DISPENSARY AND TO THE EXMOUTH COTTAGE HOSPYTAL.

For close upon ten years I have been accustomed to consider no routine examination of the urine complete without microscopical investigation of the centrifuged deposit, and realising that useful information might subsequently be derived, the results of examination have been carefully noted in a book specially tabulated for the purpose. Observations of this kind taken by the man engaged in general practice have this merit, that they are not, as a rule, limited to one observation of the patient, but may often be repeated at intervals stretched over a considerable number of years There is thus at his disposal which the consultant must of necessity lack- $\mathrm{viz}_{s}$, a constant means of testing the prognostic value of any given sign or symptom.

With regard to the subject of this communication much has been written, and much difference of opinion expressed as to the interpretation which should be given to the presence of renal tube casts in the urine of those in whom there is no other evidence of renal disease, whilst in the case of an established nephritis no one disputes the pathological import of their presence. Here, however, the teaching of the older text-books on medicine has nowadays to be modified, for Sahli, in his fourth edition of " Diagnostic Methods," " says as follows: "Little can be claimed for the diagnostic importance of the different varieties of castsgranular and waxy (as opposed to hyaline and epithelial) were formerly supposed to indicate a chronic process; but the idea was wrong. Any and all varieties of casts may be found in every type of nephritis, and even an amyloid kidney does not produce any distinctive type of cast." I would emphasize the words any and all varieties of easts may be found, for so frequently one has heard it laid down that the hyaline cast is a matter of small consequence in the deposit. Napoleon Boston, who has written extensively on urinary analysis, is in agreement with Leube and Purdy, who state that urine containing hyaline casts either contains albumin or has recently contained it, even though in small amounts. Saundby, ${ }^{3}$ writing at a time when the centrifuge was coming more into general use, considered that the findings of that machine were such as to cause unnecessary misgivings, and he sounded a warning note as to attaching too much importance to reports on urinary analyses conducted in research laboratories where the centrifuge was in routine use. The article was published at a time when I had not the data by which to support or refute the statement, but during the nine years which have followed the constant examination of the deposit brings out, at any rate from my own researches, two salient facts : 1 . The large number of cases (74 out of 134) in which, when the presence of casts was first noted, the urine failed to react to the routine tests for albumin. 2. Whilst the majority of these cases were examined in a condition of temporary or permanent morbidity, it is nevertheless impossible from their subsequent behaviour to dissociate the presence of casts from the particular affection in question.

In support of these statements I propose to analyse briefly the 74 cases, the urine of whom when first examined was

I A paper read before the Devon and Exeter Medico-Chirurgical Society on April 11th, 1913. found to contain casts but failed to give the reaction for albumin. Of these there are-

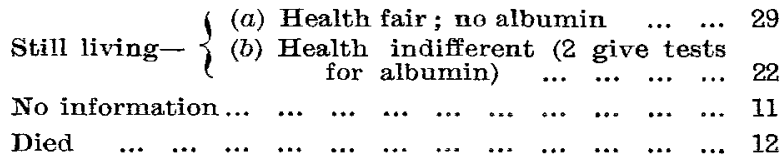

Of the 29 cases in division (a), of those still living and whose health we can presume to be in fair although in the majority in by no means sound health, there were of-

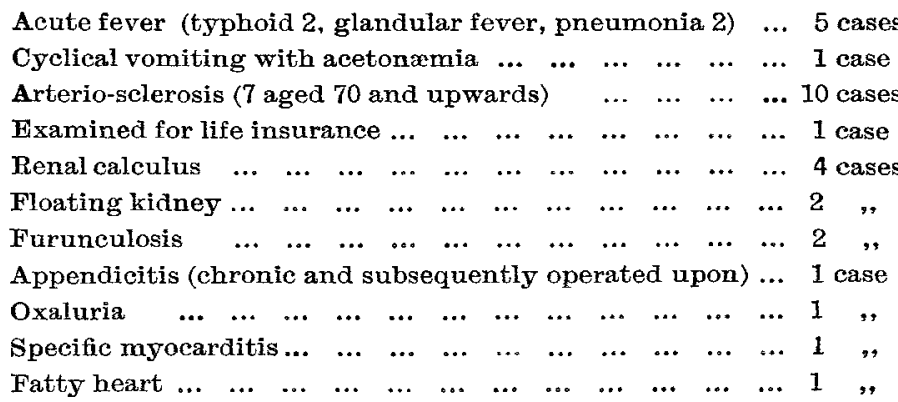

The acute cases call for little comment, the casts appearing at an early stage of the illness, and doubtless symptomatic of cloudy swelling in the kidney. In the case of cyclical vomiting (child aged 4) the casts were of the hyaline variety, and disappeared with the fall of the temperature and cessation of vomiting. The ferric chloride test was positive. The arterio-sclerotic cases will be considered later. Four cases are for convenience classified as renal calculus, but were not all verified by $X$ ray or operation. The symptoms present, however, pointed strongly, if not to the presence of an actual stone, at least to collections of crystals extensive enough to produce colic. Hyaline casts have been particularly noted in a sediment rich in crystals of calcium oxalate, and have disappeared pari passu with the latter. Casts were noted in two cases of floating kidney in this series. In addition the sediment in one case showed abundant epithelial cells of renal type. At the operation undertaken for replacement and fixation a large bæmatoma was found beneath the true capsule. Microscopical evidence of renal irritation has been my experience in a majority of these cases, and brings me to advise a belt or operation for replacement wherever I find a genuine movable kidney.

The two patients suffering from boils were both men over 40 years of age; their urine contained a few hyaline casts and cylindroids. Possibly these are gouty men whose kidneys are beginning to show wear and tear from elimination of an excess of waste products. The case of appendicitis refers to a man aged 46, who was in a state of general ill-health owing to a chronic inflammatory condition with adhesions. Qperation restored him to fair health, but I have since had no opportunity of testing his urine. The two remaining cases consulted me for cardiac conditions: the first with symptoms of arrhythmia gave a history of syphilis; the second, a stout middle-aged lady, suffered also from "irregular gout."

Class $(b)$ Health indifferent:-
Valvular cardiac disease $\ldots \ldots$ cases

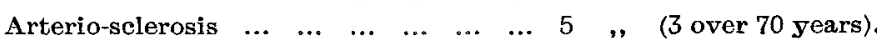

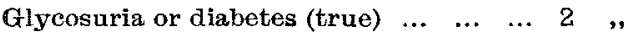

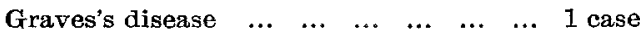
$\begin{array}{llllllllll}\text { Renal colic } & \ldots & \ldots & \ldots & \ldots & \ldots & \ldots & \ldots & 1 & \end{array}$
$\begin{array}{lllllllllll}\text { Seiatica } & \ldots & \ldots & \ldots & \ldots & \ldots & \ldots & \ldots & \ldots & 1 & \end{array}$
$\begin{array}{lllllllll}\text { Old nephritis } \ldots & \ldots & \ldots & \ldots & \ldots & \ldots & \ldots & 1 & \end{array}$
$\begin{array}{lllllllllll}\text { Asthma } & \ldots & \ldots & \ldots & \ldots & \ldots & \ldots & \ldots & \ldots & 1 & ,\end{array}$
Cardiac (non-valvular)...
$\begin{array}{llllllllll}\text { Gout } \ldots & \ldots & \ldots & \ldots & \ldots & \ldots & \ldots & \ldots & \ldots & 1 \text { case }\end{array}$

In this series two of the cases occasionally show a trace of albumin.

The five cases of cardiac valvular disease were patients of middle or advanced age, and the majority showed evidence of arterial degeneration. No one of them showed signs of backward pressure, and possibly in some of them the cast association was primarily in the kidney. Of those labelled arterio-sclerosis three out of five had passed their seventieth year. The remaining two were aged 62 and 69 respectively, 